\title{
Bistable Microwave Oscillator Based on Nonlinear Magnetostatic Wave Transmission Line
}

\author{
Y.K. Fetisov \\ Moscow Institute of Radioengineering, Electronics and Automation, pr. Vernadskogo 78, \\ Moscow 117454, Russia
}

\begin{abstract}
A microwave oscillator containing a GaAs amplifier, a magnetostatic spin wave (MSW) signal-to-noise enhancer (SNE) used as a nonlinear transmission line, directional couplers, and a variable attenuator has been designed and investigated. It was shown, that nonlinearity of the SNE and amplifier resulted in a bistability in the system: two different levels of oscillation power correspond to one attenuator setting. The oscillator was switched on under action of external short low power microwave pulses.
\end{abstract}

\section{INTRODUCTION}

Microwave feedback oscillators containing an amplifier and a magnetostatic spin wave (MSW) transmission line or resonator in the feedback loop have been intensively investigated because of two reasons. Firstly, tunability of MSW characteristics allows electrical changing of the oscillator frequency in the $2-20 \mathrm{GHz}$ frequency band [1]. Secondly, high slope of the MSW phase-frequency characteristic provides low phase noise of the oscillator [2]. This paper for the first time demonstrates a possibility to realize a bistable behavior of the microwave feedback oscillator using high nonlinearity of the MSW transmission line

\section{MSW OSCILLATOR DESIGN}

The schematic diagram of the MSW oscillator is shown in Fig.1. The oscillator contained a microwave amplifier G, two directional couplers B and C, a nonlinear MSW transmission line SNE, a narrow band microwave filter YIG, and a variable attenuator $\mathrm{A}$ all connected in the feedback loop. The GaAs monolithic amplifier had a low signal gain coefficient $G=31 \mathrm{~dB}$ in the $f=2.5-6 \mathrm{GHz}$ frequency band and maximum output power $P_{s}=0.5 \mathrm{~W}$. Coupling coefficients of the input and output couplers were equal to $B=-16 \mathrm{~dB}$ and $C=-22 \mathrm{~dB}$, respectively. The tunable yttrium-iron-garnet (YIG) sphere filter had a passband of $\Delta f=40 \mathrm{MHz}$ and insertion loss $-5 \mathrm{~dB}$ at the central frequency $f=3.23 \mathrm{GHz}$. The variable attenuator provided attenuation in the feedback loop $A=0-120 \mathrm{~dB}$. A MSW signal-to-noise enhancer (SNE) described in detail in [3], was used as the nonlinear microwave transmission line. The SNE contained a $30 \mu \mathrm{m}$ thick monocrystalic YIG film magnetized with a DC tangential field $H=310$ Oe and a $12 \mathrm{~mm}$ long and $50 \mu \mathrm{m}$ wide microstrip transducer deposited on an alumina substrate. The SNE transmission characteristic is shown in Fig.2. Within the operational frequency band $f=3-3.4 \mathrm{GHz}$ it provided high insertion loss $L=-23 \mathrm{~dB}$ for low power input signals $P<1 \mathrm{~mW}$ and low insertion loss $L=-12 \mathrm{~dB}$ for high power signals $P>0.4 \mathrm{~W}$.

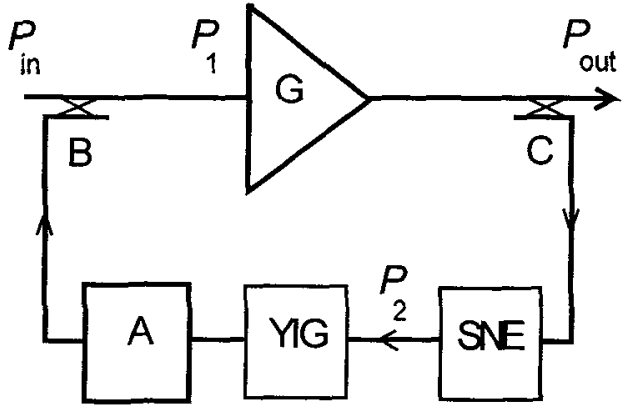

Figure 1: Schematic diagram of MSW oscillator.

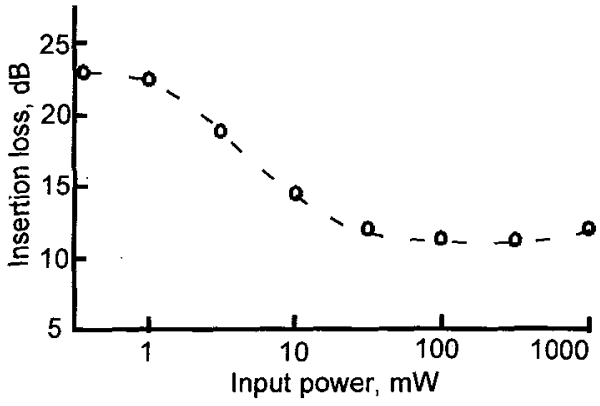

Figure 2: Transmission characteristic of SNE. 


\section{EXPERIMENTAL RESULTS AND DISCUSSION}

The oscillation in the feedback loop arises at the filter passband frequency $(f=3.23 \mathrm{GHz}$ in our case) when total amplification of the signal $G$ becomes higher than total loss in the loop: $G L R \geq 1$, where $R$ includes loss in the attenuator $A$, loss in both couplers $(1-B)(1-C)$, loss in the YIG filter, and loss in all connection lines. With that, the level of microwave power in the loop is determined by specific nonlinear characteristics of the amplifier and the SNE.

The Fig. 3 shows dependence of the power $P_{2}$ at the SNE output versus power $P_{1}$ at the amplifier input for consequently connected amplifier and SNE taken separately. The region I corresponds to the linear regime of the amplifier operation and high insertion loss of the SNE. In the region II the amplifier remains in the linear operation regime while the insertion loss of the SNE is decreasing. The region III corresponds to transaction of the amplifier to saturation and low insertion loss of the SNE. As a result, one obtains a nonlinear transmission characteristic $P_{2}=F\left(P_{1}\right)$ (line 1 in Fig.3) having a concave region with $\partial^{2} P_{2} / \partial P_{1}^{2}>0$. From the other hand, consideration of linear elements of the loop gives that microwave powers are connected by the relation $P_{2}=(1 / R) P_{1}$. Straight lines 2,3 , and 4 in Fig. 3 correspond to different values of insertion loss $R_{2}>R_{3}>R_{4}$.

With decreasing of the loss in the loop the oscillation starts when the slope of the straight line becomes equal to the slope of characteristic $P_{2}=F\left(P_{1}\right)$ in the low power region $\left(P_{1} \rightarrow 0\right)$. At the value $R_{2}$ the power of microwave signal at the amplifier input increases abruptly up to the level $P_{1}^{\prime}$ that corresponds to the output power $P_{\text {out }}=G\left(P_{1}^{\prime}\right) C$. Further decrease of the loss results in corresponding increase of power in the loop while output power $P_{\text {out }}$ remains nearly constant because of saturation of the amplifier.

With increasing of the loss the oscillation exists until the straight line becomes tangent to the characteristic $P_{2}=F\left(P_{1}\right)$. At the value $R_{3}$ the power of output microwave signal decreases abruptly up to the noise level. Thus, in the region of the loss variation $R_{3}<R<R_{2}$ two different values of microwave power correspond to one value of insertion loss $R$.

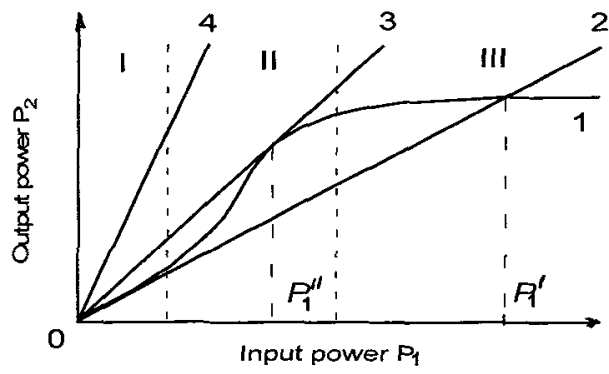

Figure 3: Relations between microwave powers $P_{1}$ and $P_{2}$ in the MSW oscillator loop.

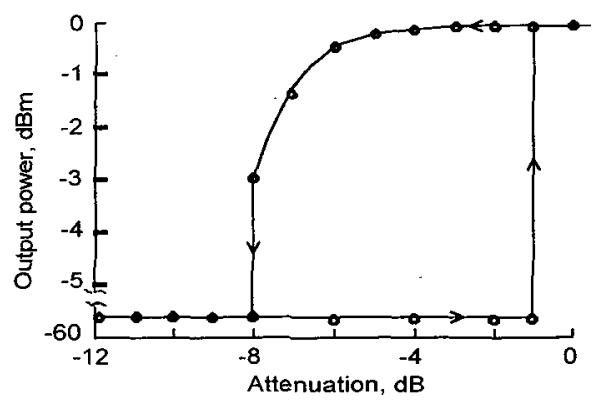

Figure 4: Dependence of MSW oscillator power $P_{\text {out }}$. versus setting of attenuator $A$.

Fig. 4 shows measured dependence of the oscillator output power $P_{\text {out }}$ versus setting of the attenuator $A$ in the feedback loop. The arrows show variation of the power with decreasing and increasing of attenuation. In accordance with theoretical predictions, two different levels of output power have been observed in the region of attenuation $-8 \mathrm{~dB}<A<-1 \mathrm{~dB}$. Output power increased abruptly at $A=-1 \mathrm{~dB}$ with decrease of attenuation and decreased abruptly at $A=-8 \mathrm{~dB}$ with increase of attenuation.

Hard excitation of the MSW oscillator being in the bistable condition has been realized as well. The oscillator was turned on by applying an external microwave pulse with carrier frequency $f \cong 3.2 \mathrm{GHz}$, duration $\tau \geq 25 \mathrm{~ns}$, and peak power $P_{\text {in }} \geq 1.8$ $\mathrm{mW}$ to the input coupler $\mathrm{B}$. Minimum energy of the pulse required to start the oscillation was equal to $W=\tau P_{\text {in }} \approx 5 \cdot 10^{-11} \mathrm{~J}$.

\section{CONCLUSION}

Thus, our experiments demonstrated existence of bistability in the microwave feedback oscillator containing a nonlinear MSW signal-to-noise enhancer. Output power of the oscillator being in the bistable condition depends on the preceding history of the system and the setting of attenuator in the feedback loop. The oscillator can be turned on by short external microwave pulses.

\section{Acknowledgements}

The author is grateful to Prof. C. E. Patton and Prof. P. Kabos for support and useful discussions.

\section{References}

[1] Ishak W., Electron. Lett., 19 (1983) 930-931.

[2] Dunaev S. N., Fetisov Y. K., Tokarev V. G. and Vasil'ev V. P., Sov. J. of Commun. Tech. and Electr. 37 (1992) $65-70$.

[3] Stitzer S. S. and Emtage P. R., Circuits, Systems and Signal Processing 4 (1985) 228-252. 